\title{
Exploring Mixed-Scale Gesture Interaction
}

\author{
Barrett Ens \\ University of South Australia \\ Adelaide, Australia \\ barrett.ens@unisa.edu.au \\ Pourang Irani \\ University of Manitoba \\ Winnipeg, Canada \\ pourang.irani@cs.umanitoba.ca
}

\author{
Aaron Quigley \\ University of St. Andrews \\ St. Andrews, Scotland \\ aquigley@st-andrews.ac.uk
Thammathip Piumsomboon
University of South Australia
Adelaide, Australia
pourang.irani@cs.umanitoba.ca

\author{
Hui-Shyong Yeo \\ University of St. Andrews \\ St. Andrews, Scotland \\ hsy@st-andrews.ac.uk
}

\author{
Mark Billinghurst \\ University of South Australia \\ Adelaide, Australia \\ mark.billinghurst@unisa.edu.au
}

\begin{abstract}
This paper presents ongoing work toward a design exploration for combining microgestures with other types of gestures within the greater lexicon of gestures for computer interaction. We describe three prototype applications that show various facets of this multi-dimensional design space. These applications portray various tasks on a Hololens Augmented Reality display, using different combinations of wearable sensors.
\end{abstract}

\section{CCS CONCEPTS}

- Human-centered computing $\rightarrow$ Mixed / augmented reality;

\section{KEYWORDS}

microgestures, gesture interaction, augmented reality

\section{ACM Reference Format:}

Barrett Ens, Aaron Quigley, Hui-Shyong Yeo, Pourang Irani, Thammathip Piumsomboon, and Mark Billinghurst. 2017. Exploring Mixed-Scale Gesture Interaction. In Proceedings of SA '17 Posters. ACM, New York, NY, USA, 2 pages. https://doi.org/10.1145/3145690.3145740

\section{INTRODUCTION}

Computer interfaces have often made successful use of gestures to allow rich and intuitive interaction. The strategy of mimicking our everyday interactions with real-world objects allows us to map highlevel mental models onto appropriate sequences of physical actions [Buxton 1986]. For example, mouse users apply a combination of mouse movements and clicking gestures to select or move data objects. More recently, touch screens allow tapping and swiping gestures to be made with bare hands, which may useful when a mouse and physical desktop are not available. In next-generation Augmented Reality (AR) interfaces, gesture interaction can closely resemble real-world interaction, since users can point at [Bolt 1980] or grasp virtual objects directly overlaid on their environment.

While such "natural" interactions can be highly appealing for AR applications, "in-air" gestures are prone to disadvantages such as

(C) ACM, 2014. This is the author's version of the work. It is posted here by permission of ACM for your personal use. Not for redistribution. The definitive version will be published in SA '17 Posters, November 27-30, 2017, Bangkok, Thailand

(c) 2017 Copyright held by the owner/author(s).

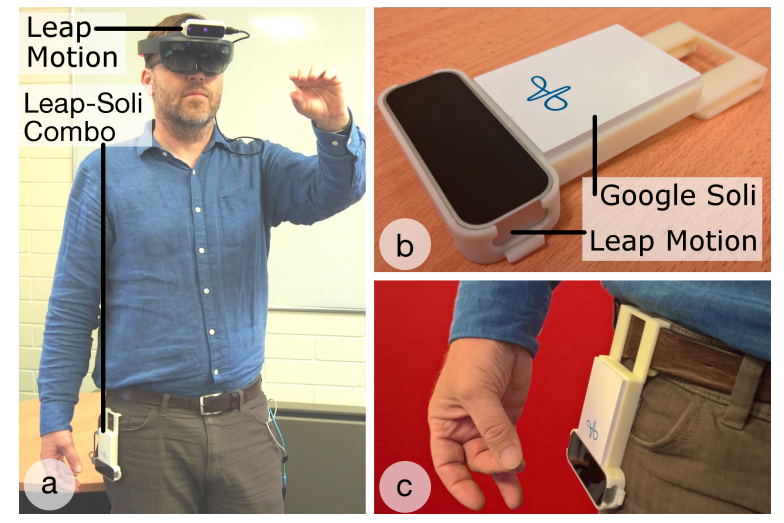

Figure 1: a) A belt sensor configuration (b, c) for use with a Hololens combines a Leap and Soli sensors.

fatigue, imprecision, and social awkwardness. Fatigue is caused by interactions that require the arms to be extended from the body for a prolonged period [Hincapié-Ramos et al. 2014]. Precise interaction is known to be difficult without the aid of a haptic surface, limiting practical applications. Social awkwardness may arise in some social contexts, when gestures attract attention from observers who do not have a complete picture of what the user is doing.

To address these concerns, researchers have explored the use of microgestures, minute gestures performed by the hands or fingers [Wolf et al. 2011], for computer interaction. Microgestures rely on sophisticated sensing methods that are now capable of detecting fine-scale hand motions. Such methods include computer vision techniques for articulated hand-tracking [Rehg and Kanade 1994] and sub-millimeter radar [Lien et al. 2016], which gives high temporal resolution. Microgestures have primarily been explored on their own, independent of other types of gestures [Wolf et al. 2011]. In contrast, this work builds toward a design space exploration for mixing microgestures within the greater gestural lexicon [Ens et al. 2016]. Our work is among the first to combine recent sub-millimeter radar technology, which is capable of tracking very fine motions, with other sensors for gesture tracking.

We present Counterpoint, an ongoing design exploration for gestural AR interaction that combines interaction on multiple scales. Using multiple wearable sensors (fig. 1), we are currently developing several prototype implementations that explore various ways to combine gestures. For instance, macro-scale grasping and reaching gestures can allow quick and intuitive direct manipulation of 
(a)

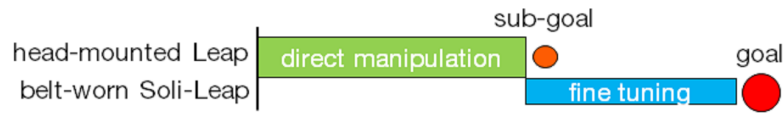

(b)
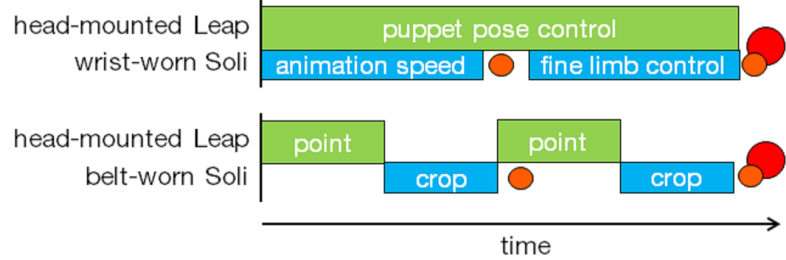

Figure 2: Graphical representations of interactions from our prototypes: a) docking task, b) virtual puppetry and c) insitu video editing.

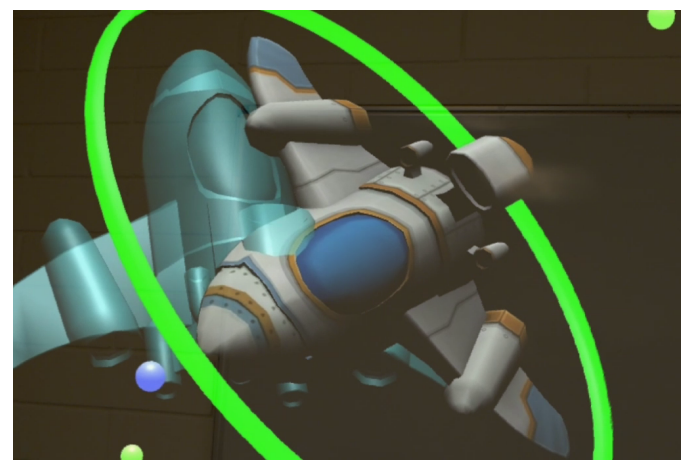

Figure 3: Spaceship docking application as viewed through the Hololens AR display.

virtual objects, while microgestures allow fine precision of object placement. Overall, we focus on allowing precise interaction and reducing fatigue, with smooth transitions between macro and micro gesture scales.

\section{PROTOTYPE APPLICATIONS}

Here we discuss three prototype applications under development. These applications demonstrate a range of ways that microgestures can be interleaved with other types of gestures into longer interaction phrases. Each application combines multiple sensors, used with a Hololens AR display [Microsoft 2017]. An overview of these applications is shown in fig. 2. Various elements of the design space are emphasized below using italics.

The first application (2a) demonstrates precise object manipulation through a docking task. A head-worn Leap [Leap Motion 2017] sensor (fig. 1a) can detect grasping and manipulation gestures. Whereas such gestures are prone to fatigue, a belt-worn Soli+Leap configuration (fig. 1b, c) can provide a second input mode with the arm down [Liu et al. 2015]. The Leap provides hand pose information to map six thumb sliders onto the tips and sides of the first three fingers, allowing motion sensed by the Soli [Google 2017] to provide continuous control of the object's three axes of translation and rotation (fig. 3), respectively.

The second application controls a virtual puppet (fig. 4b). As in the first example, the head-mounted Leap provides coarse control of the puppet's pose. However, in this case, microgestures are used

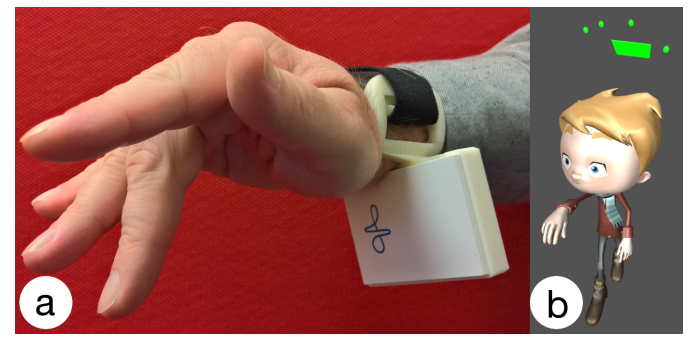

Figure 4: a) A wrist-worn Soli sensor provides precise control for aspects of a virtual puppet (b).

simultaneously by the same hand. A wrist-mounted Soli sensor provides precise control over various continuous puppet actions such as limb motions or the speed of preset animations, which can be triggered by discrete finger poses.

The third example under development demonstrates a tool for in-situ cropping and editing of short videos (fig. 4b), which may be taken with an AR display's camera. This example demonstrates bimanual interaction; the head-mounted sensor can detect coarse pointing gestures, in relation to a virtual video clip, while various pre-trained motion gestures are recognized by a belt-worn Soli sensor. For instance, two fingers can imitate scissors making a "cut" gesture to crop a segment.

These prototypes demonstrate a sample of various dimensional mappings from a complex gesture design space. In future work, we will elaborate on the taxonomy of the first such design space to include microgestures within the greater gestural lexicon. We plan to continue development of these and other applications to provide a thorough exploration of this space.

\section{REFERENCES}

Richard a Bolt. 1980. "Put-that-there": Voice and gesture at the graphics Interface. Proceedings of the 7th annual conference on Computer graphics and interactive techniques - SIGGRAPH '80 (1980), 262-270. https://doi.org/10.1145/800250.807503

Bill Buxton. 1986. Chunking and phrasing and the design of human-computer dialogues. Proceedings of the IFIP World Computer Congress 1986 (1986), 475-480.

Barrett Ens, Ahmad Byagowi, Teng Han, Juan David Hincapié-Ramos, and Pourang Irani. 2016. Combining Ring Input with Hand Tracking for Precise, Natural Interaction with Spatial Analytic Interfaces. In Proceedings of the 2016 Symposium on Spatial User Interaction - SUI '16. ACM Press, New York, New York, USA, 99-102. https://doi.org/10.1145/2983310.2985757

Google. 2017. Project Soli. (2017). https://atap.google.com/soli/

Juan David Hincapié-Ramos, Xiang Guo, Paymahn Moghadasian, and Pourang Irani. 2014. Consumed Endurance: A metric to quantify arm fatigue of mid-air interactions. Proceedings of the 32nd annual ACM conference on Human factors in computing systems - CHI '14 (2014), 1063-1072. https://doi.org/10.1145/2556288.2557130

Leap Motion. 2017. Leap Motion. (2017). https://www.leapmotion.com/

Jaime Lien, Nicholas Gillian, M. Emre Karagozler, Patrick Amihood, Carsten Schwesig, Erik Olson, Hakim Raja, and Ivan Poupyrev. 2016. Soli. ACM Transactions on Graphics 35, 4 (jul 2016), 1-19. https://doi.org/10.1145/2897824.2925953

Mingyu Liu, Mathieu Nancel, and Daniel Vogel. 2015. Gunslinger. In Proceedings of the 28th Annual ACM Symposium on User Interface Software \& Technology - UIST '15. ACM Press, New York, New York, USA, 63-71. https://doi.org/10.1145/2807442. 2807489

Microsoft. 2017. The leader in Mixed Reality Technology | HoloLens. (2017). https: //www.microsoft.com/en-us/hololens

J M Rehg and T Kanade. 1994. Visual Tracking of High DOF Articulated Structures: An Application to Human Hand Tracking. Proceedings of the European Conference on Computer Vision (ECCV) 801 (1994).

Katrin Wolf, Anja Naumann, Michael Rohs, and Jörg Müller. 2011. A taxonomy of microinteractions: Defining microgestures based on ergonomic and scenarioDependent requirements. Lecture Notes in Computer Science (including subseries Lecture Notes in Artificial Intelligence and Lecture Notes in Bioinformatics) 6946 LNCS, PART 1 (2011), 559-575. https://doi.org/10.1007/978-3-642-23774-4_45 